\title{
Appendicular Stump Closure by Polymer Clip vs Endoloop in Laparoscopic Appendectomy
}

Vishal P Bhabhor

\begin{abstract}
Introduction: Several techniques are used to close the appendicular stump during laparoscopic appendectomy. This is especially important in developing countries where resources for training with endoloop are insufficient, and the simplicity of application of polymer clips can enable easy acceptance of laparoscopic appendectomy as a method in the treatment of acute appendicitis.

Aim: The aim of the study was to compare results of appendicular stump closure by polymer clip and endoloop with reference to

- Days of hospitalization,

- Infection rate, and

- Ease of application

Materials and methods: This is a prospective study of 70 cases of laparoscopic appendectomy operated in the Department of General Surgery, SSG Hospital, Vadodara, from October 2016 to October 2017 (35 patients in each group i.e., polymer clip application group and endoloop application group). Operative data were recorded, and the patients were followed up accordingly. Independent assessors were assigned to obtain days of hospitalization, infection rate, ease of application, and other secondary outcomes.

Results: It is more feasible for surgeons to use polymer clip than endoloop to close appendicular stump as per the surgeon's opinion taken after each surgery. However, days of hospitalization and postoperative complication rates were not statistically significant in both groups.

Conclusion: Closure of appendix stump with a polymer clip is a simple and safe method in laparoscopic appendectomy and is comparable with other methods of appendicular stump closure.

Keywords: Appendectomy, Ease of application, Endoloop, Polymer clip.

World Journal of Laparoscopic Surgery (2019): 10.5005/jp-journals-10033-1374
\end{abstract}

\section{INTRODUCTION}

Laparoscopic appendectomy is superior or comparable to open appendectomy in terms of several surgical outcome measures for both uncomplicated and complicated appendicitis across most illness severity groups. Thus, laparoscopic appendectomy may be the preferred technique, irrespective of appendicitis diagnosis or disease severity. ${ }^{1}$ Several techniques are used to close the appendicular stump during laparoscopic appendectomy.

The most commonly used surgical methods are associated with the use of endoloop ligature, laparoscopic staplers, metal or polymer clips, or application of purse-string suture with the invagination of the base of the appendix into the cecum, as in the classic surgery. ${ }^{2}$

However, the application of the endoloop demands dexterity and short training, while polymer clips may be more advantageous to use due to their easy of application and low cost. ${ }^{3}$

This is especially important in developing countries where resources for training with endoloop are insufficient, and the simplicity of application of polymer clips can enable easy acceptance of laparoscopic appendectomy as a method in the treatment of acute appendicitis. ${ }^{3}$

The objective of this study was to evaluate the technical feasibility and eventual advantages of this way of securing the base of the appendix.

\section{Materials and Methods}

This is a prospective study of 70 cases of laparoscopic appendectomy operated in the Department of General Surgery, SSG Hospital,
Department of General Surgery, Baroda Medical College, SSG Hospital, Maharaja Sayajirao University of Baroda, Vadodara, Gujarat, India

Corresponding Author: Vishal P Bhabhor, Department of General Surgery, Baroda Medical College, SSG Hospital, Maharaja Sayajirao University of Baroda, Vadodara, Gujarat, India, Phone: +91 7600543427, e-mail: bhabhorvishal33@gmail.com

How to cite this article: Bhabhor VP. Appendicular Stump Closure by Polymer Clip vs Endoloop in Laparoscopic Appendectomy. World J Lap Surg 2019;12(2):64-67.

Source of support: Nil

Conflict of interest: None

Vadodara from October 2016 to October 2017 (35 patients in each group, i.e., polymer clip application group and endoloop application group).

Patients were included in the study after proper clinical examination, laboratory findings, and ultrasonographic evidence of acute appendicitis in the outpatient department and emergency. The surgeon was the deciding method for closing appendicular stump by viewing the appendicular base and its diameter (by endoloop or by polymer clip).

All laparoscopic appendectomy was done by a consultant surgeon of different units of Surgery Department of SSG Hospital, Vadodara. Patients scheduled for laparoscopic appendectomy were administered antibiotics intravenously in the form of single-dose ceftriaxone just before the start of the surgery.

(c) The Author(s). 2019Open Access This article is distributed under the terms of the Creative Commons Attribution 4.0 International License (https://creativecommons. org/licenses/by-nc/4.0/), which permits unrestricted use, distribution, and non-commercial reproduction in any medium, provided you give appropriate credit to the original author(s) and the source, provide a link to the Creative Commons license, and indicate if changes were made. The Creative Commons Public Domain Dedication waiver (http://creativecommons.org/publicdomain/zero/1.0/) applies to the data made available in this article, unless otherwise stated. 


\section{Operative Technique}

Pneumoperitoneum was established using a Veress needle inserted from the infraumbical region. The first $10 \mathrm{~mm}$ trocar was introduced in the abdomen just below the umbilicus.

The second $5 \mathrm{~mm}$ trocar was placed just above the pubic symphysis and the third trocar of $5 \mathrm{~mm}$ in the left lower abdomen below the level of the umbilicus in the line of the anterior axillary fold. From $10 \mathrm{~mm}$ scope, $30^{\circ}$ telescope was inserted and from $5 \mathrm{~mm}$ port dissection was performed.

After viewing the abdominal organs and confirming the diagnosis of acute appendicitis, the appendix was mobilized and skeletenized by cutting its mesoappendix using a harmonic scalpel or cautery. Further procedure to apply clips or endoloop was decided by the operating surgeon.

\section{For Polymer Clip Application}

A $30^{\circ}$ telescope was inserted from $5 \mathrm{~mm}$ scope, and from $10 \mathrm{~mm}$ trocar polymer clip applicator was inserted. The appendicular base was seen intraoperatively and an idea about diameter was made by the surgeon. If the diameter of the appendicular base was less than or equal to $10 \mathrm{~mm}$ approximately than it is easily closed by polymer clips. First clip was applied at the base of the appendix using clip applicator (Fig. 1), and a second clip was applied above the first clip using clip applicator. Then the appendix was cut with a harmonic scalpel or scissor above the second clip, and it was removed from $10 \mathrm{~mm}$ port. Ethicon HEM-O-LOK clip (polymer clip, Fig. 2) was used in this study. Polymer clip which was slipped or not covering appendicular stump was considered as a wasted clip.

\section{For Endoloop Application}

Three endoloops were applied on appendicular base (Fig. 3) and the appendix was cut between the second and third loop by harmonic scalpel or scissors. The appendix was removed by a 10 $\mathrm{mm}$ port. Endoloop was made outside from chromic catgut and introduced by knot pusher from the trocar. Endoloop which was slipped and not covering appendicular stump was considered as wasted endoloop. In both groups, $10 \mathrm{~mm}$ port incision was closed with port vicryl and other port incisions were closed by ethilon 2-0. After the appendectomy appendix was sent for histopathologic examination in both groups.

Endoloop and clips are equally cost-effective, but the clip appears to be simpler in handling than the endo-loop and, like the stapler, offers closing, and cutting the appendix before dissecting

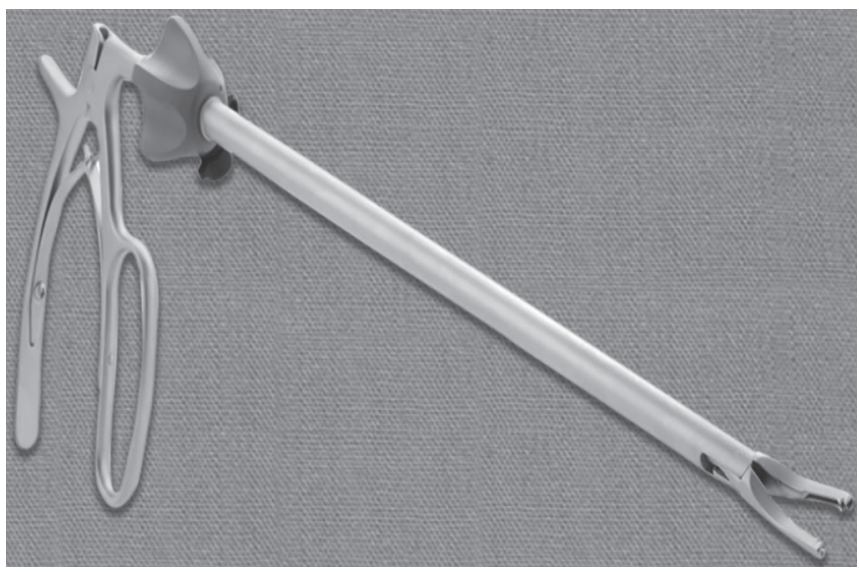

Fig. 1: Polymer clip applicator the mesoappendix. However, only appendices up to $16 \mathrm{~mm}$ in diameter can be clipped, a disadvantage not shared with the loop. ${ }^{4}$

Ease of application for endoloop and polymer clips was decided by the surgeon's opinion that was obtained by giving surgeon opinion form having options mentioned below

- Poor

- Good

- Excellent.

\section{Ease of Application}

It was feasibility to close appendicular stump by polymer clip and endoloop, which was decided by the surgeon.

Ease of application measures feasibility for the surgeon that includes the time taken for closing appendicular stump, the proper closure of appendicular stump, and handling of endoloop and polymer clip intraoperative, which was decided by the surgeon; and he gives opinion accordingly. Time taken for closure of appendicular stump was time taken after skeletonization of appendix up to the closure of appendicular stump. After all this, considering the surgeon had given his opinion regarding "ease of application" in this study.

\section{Postoperative Care and Follow-up}

Intravenous antibiotic (injection ceftriaxone $1 \mathrm{~g} 12$ hourly) was given for 1 day than patients shifted on an oral antibiotics (tablet cefixime $200 \mathrm{mg}$ 1BD). The first dressing was done after 48 hours, and further dressing was done only when there was soakage.

Discharge from the wound was sent for culture and sensitivity test and then patients treated accordingly. Temperature more than $98.6^{\circ} \mathrm{F}$ was considered as fever in this study. If a patient had a fever or wound discharge, then the total count was done. Ultrasonography (USG) was done in all cases after 48 hours. If the total count was more than $11,000 / \mathrm{mm}^{3}$, then it was considered as wound infection.

Patients having no complaint of fever or wound discharge, then they were discharged after 48 hours with 5 days of oral antibiotic (tablet cefixime $200 \mathrm{mg}$ 1BD); the same for both groups. The patient was not discharged when there were complaints, such as fever and wound discharge. Patients having fever given oral paracetamol (500 $\mathrm{mg}$ ) as and when required.

Patients having wound discharge were given antibiotics according to culture and sensitivity report and sutures were removed after control of infection. If a patient had no complications,

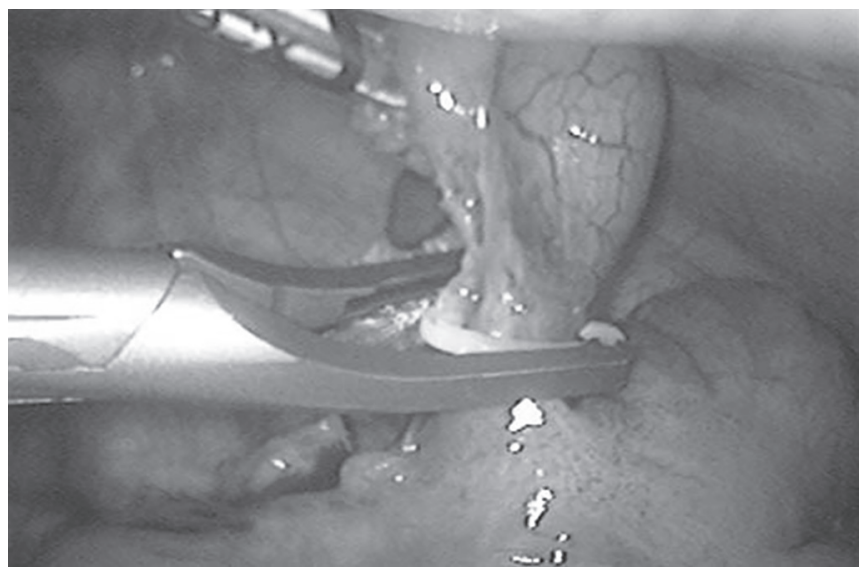

Fig. 2: Polymer clips 
then sutures were removed on the seventh postoperative day in the follow-up.

Patients had come for follow-up in the outpatient department after 1 week, 2 weeks, and 1 month of surgery. Ultrasonography was done 1 month after surgery in all cases. In follow-up, the patient was clinically examined for complications, such as wound discharge, swelling over the local site, tenderness on the abdomen, and fever.

\section{Statistical Analysis}

Data analysis was performed using MedCalc version 17.9.5 software. Categorical variables were analyzed with the Chi-squared test and continuous variables were analyzed with " $t$ " test. Values were reported as mean \pm standard deviation or median (extremes) or percentages as and when required. $p$ value of less than 0.05 was considered significant.

\section{Results}

A total of 70 patients with laparoscopic appendectomy were included in this study. Adequacy of randomization was evident from the similarity in patient characteristics in both the groups (Table 1). No protocol violations were recorded during the study.

One extra endoloop was used in four cases due to improper closure of appendicular stump. One extra polymer clip was used in

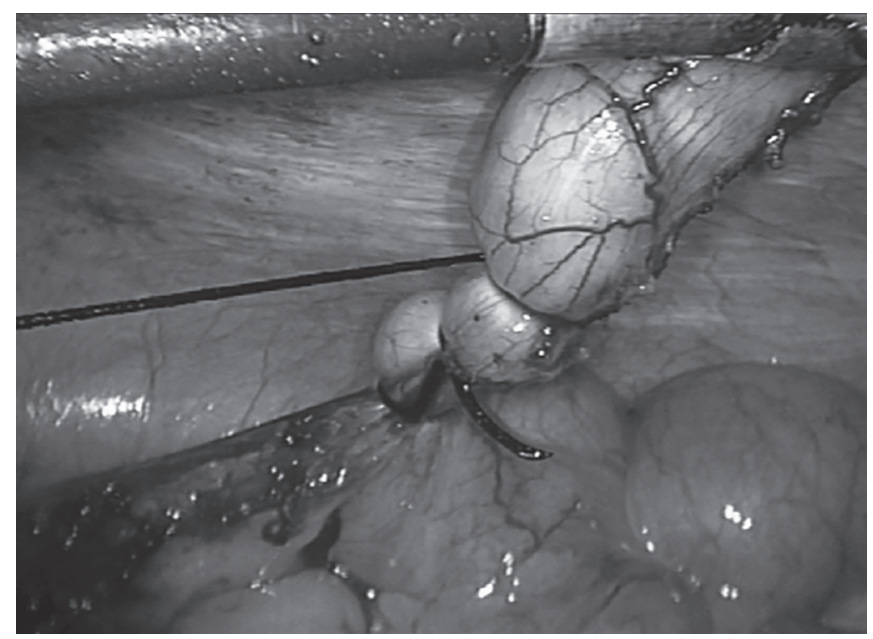

Fig. 3: Endoloop

Table 1: Comparison of present study, study done by Polat and Kinaci and study done by Osman for sex-wise distribution of patients

\begin{tabular}{lllllll}
\hline & \multicolumn{3}{c}{ Polymer clip group } & & \multicolumn{3}{c}{ Endoloop group } & \\
\cline { 2 - 5 } Different studies & Male & Female & & Male & Female & Total \\
\hline In present study & 20 & 15 & 24 & 11 & 70 \\
Polat and Kinaci & 23 & 25 & 20 & 24 & 92 \\
Şimşek & 18 & 12 & 16 & 14 & 60 \\
\hline
\end{tabular}

two cases due to improper closure of appendicular stump. These extra used polymer clips and endoloops were counted as wasted polymer clip or endoloop.

In the surgery of 21 patients, time taken for appendicular stump closure was between 10 minutes and 15 minutes and for 14 patients, it was between 15 minutes and 20 minutes in polymer clip group. In the surgery of 12 patients, the time taken for appendicular stump closure was between 10 minutes and 15 minutes, and for 23 patients it was between 15 minutes and 20 minutes in endoloop group. Surgeon had given opinion regarding the ease of application as excellent in 21 patients of polymer clip group and 12 patients of endoloop group.

The surgeon had given opinion regarding the ease of application as good in 14 patients of polymer clip group and 23 patients of endoloop group (Table 2). None of the operating surgeons given a poor opinion for endoloop group or polymer clip group. This measurement (ease of application) is statistically significant in our study ( $p$ value is 0.032 , Table 2 ).

Three patients were discharged on the fifth postoperative day in the polymer clip group, and 32 patients were discharged on the third postoperative day. Three patients were discharged on the fifth postoperative day, two patients were discharged on the seventh postoperative day, and 30 patients were discharged on the third postoperative day in endoloop group (Table 3). It is not statistically significant in our study ( $p$ value 0.144 , Table 3 ).

In three cases of endoloop group, there was a serous discharge present from the wound after the first dressing. The daily dressing was done with proper asepsis in all three cases. Culture and sensitivity for wound discharge sent and according to per the sensitivity antibiotics were started. The total count was sent but in all three cases it was below $11,000 \mathrm{~mm}^{3}$. In this study, patients having wound discharge and fever in polymer clips and endoloop groups were compared, and it was not statistically significant ( $p$ value 0.45 , Table 4 ).

Table 2: Ease of application in polymer group and endoloop group

\begin{tabular}{lccl}
\hline $\begin{array}{l}\text { Ease of } \\
\text { application }\end{array}$ & $\begin{array}{l}\text { Polymer group } \\
\text { (number of patients) }\end{array}$ & $\begin{array}{l}\text { Endoloop group } \\
\text { (number of patients) }\end{array}$ & $p$ value \\
\hline Poor & 0 & 0 & - \\
Good & 14 & 23 & 0.032 \\
Excellent & 21 & 12 & 0.032 \\
\hline
\end{tabular}

Table 3: Correlation of present study, study done by Polat and Kinaci and study done by Şimşek for hospital stay

\begin{tabular}{lllr}
\hline Different studies & $\begin{array}{l}\text { Polymer clip } \\
\text { group (in days) }\end{array}$ & $\begin{array}{l}\text { Endoloop group } \\
\text { (in days) }\end{array}$ & p value \\
\hline In present study & $3.1 \pm 0.56$ & $3.4 \pm 1.06$ & 0.144 \\
Polat and Kinaci & $1.1 \pm 0.6$ & $1.4 \pm 0.9$ & 0.061 \\
Şimşek & $2.2 \pm 1.3$ & $1.97 \pm 1.4$ & $>0.05$ \\
\hline
\end{tabular}

Table 4: Correlation of present study, study done by Polat and Kinaci and study done by Şimşek for postoperative complications

\begin{tabular}{|c|c|c|c|c|c|c|c|}
\hline \multirow[b]{2}{*}{ Different studies } & \multicolumn{3}{|c|}{ Polymer clip group } & \multicolumn{3}{|c|}{ Endoloop group } & \multirow[b]{2}{*}{$p$ value } \\
\hline & $\begin{array}{l}\text { Wound infection } \\
\text { (number of } \\
\text { patients) }\end{array}$ & $\begin{array}{l}\text { Fever (number of } \\
\text { patients) }\end{array}$ & Total patients & $\begin{array}{l}\text { Wound infection } \\
\text { (number of } \\
\text { patients) }\end{array}$ & $\begin{array}{l}\text { Fever (number of } \\
\text { patients) }\end{array}$ & Total patients & \\
\hline In present study & 0 & 3 & 35 & 3 & 2 & 35 & 0.45 \\
\hline Polat and Kinaci & 1 & 1 & 48 & 4 & 6 & 44 & 0.051 \\
\hline Şimşek & 0 & 0 & 30 & 0 & 0 & 30 & $>0.05$ \\
\hline
\end{tabular}


Ultrasonography findings of three patients in endoloop group showed minimal collection after 48 hours. In all other patients in endoloop group and polymer clip group, USG was normal. It was not statistically significant. Ultrasonography report was normal in all patients after 1 month in follow-up in endoloop and polymer clip group.

\section{Discussion}

Laparoscopic appendectomy is expected to increase gradually and become the gold standard for the treatment of acute appendicitis because laparoscopic appendectomy has advantages similar to laparoscopic cholecystectomy.

Despite differences in various laparoscopic techniques, the most important concern in laparoscopic appendectomy is the safety of the method used for the closure of appendicular stump. Therefore, there have been many defined methods with some superiority to others. Operative time, hospital stay, and postoperative complications are widely used parameters to compare the benefits of these methods.

In our conducted comparison regarding hospital stay, postoperative complications, and ease of application for appendicular stump closure polymer clip and endoloop group.

In a study by Polat and Kinaci ${ }^{5}$ in 2015 comparison done regarding operating time, hospital stay, and postoperative complications in appendicular stump closure by endoloop and polymer clip groups. In a study by Şimşek et al., ${ }^{6}$ in 2014, comparison done regarding operating time, hospital stay, cost, and postoperative complications in appendicular stump closure by endoloop and polymer clip groups.

In our study, polymer clip was found to be a more feasible technique to use for appendicular stump closure compared to endoloop as per surgeon's opinion, and it is statistically significant in our study ( $p$ value is 0.032). The opinion regarding the ease of application in our study was given by operating surgeon, and he was considered the time taken for stump closure, proper covering of appendicular stump and intraoperative handling of endoloop or polymer clips for giving his opinion.

In our study, operating time was considered as the time taken from skeletenization of the appendix to the closure of the appendicular stump. In a study by Polat and Kinaci and a study by Şimşek, operating time was taken from the insertion of the first port to the closure of appendicular stump.

Hospital stay is directly associated with postoperative complications, ${ }^{7}$ therefore, we detected similar results in these two parameters. Hospital stay and postoperative complication rates were low in polymer clip group, but it was not statistically significant for both in our study ( $p$ value for hospital stay 0.144 and postoperative complications 0.45 ).

In a study by Polat and Kinaci in 2015, hospital stay and postoperative complication rates were better in polymer clip group, but it was not statistically significant. However, the $p$ value was close to 0.05 ( 0.061 for the hospital stay and 0.051 for postoperative complications). In another study by Şimşek et al. ${ }^{6}$ in 2014, hospital stay and postoperative complication rates were not statistically significant.

As the study conducted on a small number of patients so it needs further evaluation involving a large population. In the present study, cases having intraoperative complicated appendix were not included. So to use polymer clips in such situations requires further study. The availability of polymer clip and its applicator is a major limitation for this study. The availability of a $5 \mathrm{~mm}$ telescope is also a limiting factor.

\section{Conclusion}

This is a prospective study of 70 patients operated for laparoscopic appendectomy in the Department of General Surgery, Medical College Baroda and Sir Sayajirao Gaekwad General Hospital in the period from October 2016 to October 2017.

Acute appendicitis is more common in men compared with women with a ratio of 1:1.7 (62.8\% patients were male). The use of polymer clip is well tolerated in appendicular stump closure. It is more feasible for surgeons to use polymer clip than endoloop to close appendicular stump as per the surgeon's opinion taken after each surgery.

There are early discharge and less chance of postoperative complications with the use of polymer clip in appendicular stump closure compared with endoloop. Polymer clips can be used in place of endoloop in the closure of appendicular stump with more ease and it is beneficial to the patient.

\section{Ethical Approval}

The study was approved by the institutional ethics committee.

\section{References}

1. Tiwari MM, Reynoso JF, Tsang AW, et al. Comparison of outcomes of laparoscopic appendectomy in management of uncomplicated and complicated appendicitis. Ann Surg 2011;254(6):927-932. DOI: 10.1097/SLA.0b013e31822aa8ea.

2. Strzalka M, Matyja M, Rembiasz K. Comparison of the results of laparoscopic appendectomies with application of different techniques for closure of the appendicular stump. World J Emerg Surg 2016;11:4. DOI: 10.1186/s13017-015-0060-3.

3. Delibegovic S, Matovic E. Hem-O-lok plastic clips in securing of the base of the appendix during laparoscopic appendectomy. Surg Endosc 2009;23(12):2851-2854. DOI: 10.1007/s00464-0090493-4.

4. Partecke LI, Kessler W, Patrzyk M, et al. Comparison among different closure during laparoscopic appendectomy. Surg Technol Int 2011;21:85-91.

5. Polat $Y$, Kinaci $E$. Comparison of intra corporeal knot-tying suture and hem-o-lok clip for closure of appendix stump in laparoscopic appendectomy: a retrospective study. Firat Tip Derg/Firat Med J 2015;20(2):107-109.

6. Şimşek O, Bilgin IA, Uludag S, et al. Comparison of endo-loop and polymer locking clip in ligating appendiceal stump during laparoscopic appendectomy. Eur J Endosc Laparosc Surg 2014;1(3):124-127.

7. Fischer JE, Mcdermott WV, Holmes CR, et al. Fischer's mastery of surgery. 6th ed., Wolters Kluwer; 2012. pp. 280-282. 